\title{
LABORATÓRIO DE LOGÍSTICA: TESTANDO A RELAÇÃO NÍVEL DE SERVIÇO VERSUS PREÇO DE VENDA
}

\author{
Marco Aurélio Carino Bouzada \\ Universidade Estácio de Sá (Mestrado em Administração e Desenvolvimento Empresarial) \\ Av. Presidente Vargas, $642-22^{\circ}$. Andar - Centro - Rio de Janeiro (RJ) \\ marco.bouzada@estacio.br
}

\begin{abstract}
Resumo
Será que empresas que oferecem nível de serviço logístico mais elevado alcançam mesmo maiores receitas e incorrem em custos maiores, como prega a teoria? Esta pesquisa teve como objetivo testar a relação entre nível de serviço, preço de venda e alguns indicadores de desempenho das empresas gerenciadas por participantes de um Jogo de Logística. Para testar a questão de pesquisa, foram realizadas sete análises de correlação. Os resultados (nível de serviço impactando custos e receita e preço de venda impactando receita e resultado total) foram comparados às conclusões teóricas encontradas na literatura.

Palavras-Chaves: Jogos de Empresa; Logística; Laboratório de Logística; Nível de Serviço Logístico; Preço.
\end{abstract}

\begin{abstract}
Will companies offering higher logistical service level obtain greater revenue and higher costs, like the theory says? This research aimed to test the relation among service level, selling price and some performance indicators of enterprises managed by participants of a Logistics Business Game. To test the research question, seven correlation analyses were conducted. The results (service level impacting costs and revenue and selling price impacting revenue and total result) were compared to theoretical conclusions found in the literature.

Keywords: $\quad$ Business Games; Logistics; Logistics Laboratory; Logistical Service Level; Price.
\end{abstract}




\section{INTRODUÇÃO}

Por se tratar de uma área de conhecimento muito ampla, genérica e dinâmica, a Administração de Empresas vem sofrendo diversas transformações ao longo dos últimos anos, em função das próprias pesquisas na área e das mudanças no panorama empresarial mundial. Desta maneira, tem-se tornado cada vez mais forte a necessidade de aprendizagem, treinamento e reciclagem para pesquisadores da área de Administração.

Existem diversas formas de conduzir pesquisas na área. No entanto, a metodologia de pesquisa a ser explorada neste trabalho procura inserir os envolvidos em um ambiente empresarial simulado: os Jogos de Empresas (Business Games). Tal formato, inclusive, necessita de mais pesquisas a seu respeito no país, para que possa se consolidar como uma efetiva metodologia de pesquisa no Brasil.

Mais especificamente, o "coração" desta proposta é o BR-LOG, um Jogo de Logística desenvolvido por Bouzada (2001).

Atualmente, existem alguns jogos de logística disponíveis no mercado doméstico, principalmente para fins de ensino e treinamento gerencial. No entanto, uma parcela muito reduzida deles é aplicada ao cenário brasileiro. Dentre estes, não foi possível descobrir algum em que as distâncias entre as localidades fossem verdadeiras e a disponibilidade dos modais fosse próxima da real. A maioria das aplicações utiliza localidades fictícias, o que exige uma boa capacidade de abstração. (LIMA, 2004; AZEREDO et al., 2006; CEL, 2008; BOUZADA, 2011)

Bonocielli Jr. e Lopes (2008) observam que muitos dos jogos de empresas utilizados no Brasil são adaptações de jogos importados de outros países.

Tais carências dificultam que tais opções viabilizem a efetiva pesquisa sobre Logística no Brasil. A captura de insights genéricos sobre o assunto até poderia ocorrer, mas a construção de conhecimento acerca das características específicas do mercado brasileiro ficaria prejudicada.

Além disso, a maioria dos jogos de logística existentes não contempla a possibilidade de transporte intermodal, a utilização dos modais aéreo e marítimo, a escolha da localização $\mathrm{da}(\mathrm{s})$ fábrica(s), a programação diária da produção, o transporte palletizado, o tratamento de produtos frigorificados, entre outros aspectos críticos da gestão logística de uma empresa. (LIMA, 2004; AZEREDO et al., 2006; CEL, 2008; BOUZADA, 2011)

A proposta geral, então, desta pesquisa consistiu em aplicar a metodologia intitulada "Laboratório de Logística" (BOUZADA, 2010) para testar elementos de pesquisa na área de Logística, já que ela é capaz de verificar experimentalmente diversas hipóteses de pesquisa e relacioná-las à teoria existente, confirmando-a ou refutando-a. Este é o propósito central do trabalho.

Dessa forma, teoria estaria sendo desenvolvida a partir de um método de simulação (o business game), como afirmam ser possível Davis, Eisenhardt e Bingham (2007).

Os objetivos específicos compreenderam testar:

$\checkmark \quad$ de que forma o nível de serviço logístico prestado pelas empresas no jogo é capaz de impactar as receitas, os custos e o resultado operacional destas empresas; $\checkmark \quad$ de que forma o preço de venda dos produtos praticado pelas empresas no jogo é capaz de impactar as receitas e o resultado operacional destas empresas;

$\checkmark \quad$ se o nível de serviço logístico prestado pelas empresas no jogo está alinhado com os preços de venda dos produtos praticado pelas mesmas.

\subsection{RELEVÂNCIA DA PESQUISA}

Os benefícios da metodologia de ensino aqui pesquisada - Jogos de Empresas - se tornam ainda mais marcantes em áreas que envolvam raciocínio e manuseio de variáveis quantitativas, como a Logística. (BOUZADA, 2011) 
Não há, deixe-se claro, a pretensão de sugerir que o simulador utilizado na pesquisa (o BR-LOG) é capaz de representar perfeitamente as condições do mercado brasileiro. O modelo não contempla todas as variáveis importantes e diversos parâmetros podem ir se desatualizando ao longo do tempo. Tal limitação pode ser reduzida com a adoção estrita do conceito de Laboratório de Gestão (SAUAIA, 2010), com os estudantes atualizando o modelo por meio de pesquisa aplicada conduzidas por eles em empresas reais.

Mas a existência (e o reconhecimento) de tal limitação não invalida a possibilidade de o simulador utilizado (BR-LOG) ajudar a construir conhecimento acerca dos aspectos logísticos do mercado brasileiro, ainda mais quando comparado a outros simuladores logísticos mais genéricos e/ou menos realistas.

Até porque, similares aplicações de jogos de empresas, como a relatada por Oliveira e Alves (2012), demonstraram a aderência entre o ambiente simulado em um jogo e as características encontradas no mundo real, corroborando a tendência à adoção de simuladores organizacionais como instrumentos de pesquisa na área de Gestão.

\subsection{HIPÓTESES DE PESQUISA}

Desta forma, acredita-se que os resultados deste trabalho permitiram ajudar a verificar, entre outras coisas - em um ambiente simulado, onde o erro não acarreta consequências como no mundo real - se, conforme prega a teoria (BALLOU, 1993; BESANKO et al., 2006; BOWERSOX; CLOSS, 1996; CHURCHIL, 2000; PORTER, 1980):

1. o nível de serviço é capaz de impactar positivamente os seguintes indicadores:

a. receitas;

b. custos;

c. resultado operacional;

d. retorno sobre o investimento;

2. o preço de venda é capaz de impactar os seguintes indicadores:

a. receitas;

b. resultado operacional; e

3. o nível de serviço e o preço de venda praticado pelas empresas caminham na mesma direção (níveis de serviço melhores encerrando preços maiores).

\section{REVISÃO DE LITERATURA}

No serviço ao cliente, as empresas procuram criar valores para os seus clientes através de um serviço de nível superior, com entregas mais frequentes, cumprimento de prazos e disponibilidade de mercadorias, entre outros. (GOMES; RIBEIRO, 2004) termos de:

Segundo Bowersox e Closs (1996), o nível de serviço logístico pode ser medido em

$\checkmark \quad$ disponibilidade - é a capacidade de ter estoque quando desejado pelo cliente e costuma ser medida em termos de falta de estoque, do percentual de preenchimento das ordens e da quantidade de pedidos plenamente atendidos;

$\checkmark$ desempenho operacional - pode ser medido em termos de velocidade, consistência, flexibilidade e recuperação de falhas; e

$\checkmark \quad$ confiabilidade - muito relacionada ao fornecimento de informações acuradas.

Para algumas empresas, nível de serviço logístico é o tempo necessário para entregar um pedido ao cliente; para outras, é a disponibilidade de estoque. (BALLOU, 1993)

Alguns outros indicadores, apresentados por Heskett (1971), complementam o conceito: lote mínimo de compra; proporção dos pedidos atendidos com exatidão; porcentagem de ordens atendidas dentro de um intervalo de tempo; facilidade e flexibilidade com que o cliente pode gerar o pedido. 
No ambiente operacional de hoje, praticamente qualquer nível de serviço logístico pode ser atingido se forem comprometidos os recursos necessários. Atualmente, o fator limitante é econômico e não tecnológico. Mas, na prática, o serviço perfeito seria custoso e não necessário. O serviço logístico consiste em um equilíbrio entre prioridades do serviço e custo; ou seja, o gestor logístico preocupado com o nível de serviço está sempre se perguntando se consiste em um investimento válido o custo associado a conseguir prestar serviço a um nível específico. (BOWERSOX; CLOSS, 1996)

Ballou (1993) disserta na mesma linha, colocando que um maior nível de serviço logístico custa mais que níveis menores. A solução de máximo nível de serviço deve ser demasiado cara para implementação. O custo de estabelecer certo nível de serviço deve ser contrabalançado com vendas potenciais. Em geral, quanto maior o nível de serviço, maiores serão as receitas, mas também o serão os custos. O maior lucro não é necessariamente atingido com maiores níveis de serviço.

Dessa forma, o gerenciamento logístico deve planejar e coordenar as atividades necessárias para alcançar os níveis desejáveis de serviços e qualidade, mas de forma eficiente em termos de custos. (GOMES; RIBEIRO, 2004)

Mas assim como aumentam os custos, diversos atributos do nível de serviço, como a disponibilidade de produto, a pronta entrega e o atendimento correto dos pedidos agradam ao cliente, o que pode aumentar as vendas. (BALLOU, 1993)

Gomes e Ribeiro (2004) justificam essa possibilidade, dizendo que, a partir do momento em que os produtos vão se tornando commodities, o serviço ao cliente toma importância maior na diferenciação dos produtos.

Além do nível de serviço (e da qualidade), o preço é o outro dos principais atributos que constituem o produto, de acordo com Ballou (1993).

Segundo Kotler e Armstrong (2000) é necessária uma definição clara do posicionamento do produto no mercado antes da definição do preço. É preciso sincronizar a estratégia de apreçamento com as estratégias do marketing mix (além de preço: promoção, praça e produto/serviço). Os custos também devem ser considerados, pois eles determinam o mínimo a ser cobrado pelo produto e deve incluir os custos com produção, distribuição e venda do produto.

O marketing mix das empresas (promoção, praça, produto/serviço e preço) precisa ser ajustado de acordo com as necessidades dos clientes. (BOWERSOX; CLOSS, 1996)

Uma empresa que opte pela estratégia de diferenciação deve procurar formas de se diferenciar, que normalmente são custosas como, por exemplo, oferecer um nível de serviço bem superior à concorrência. A consequência natural do custo da diferenciação é a necessidade de praticar um preço premium, capaz de compensar o custo superior. (PORTER, 1980)

De acordo com Besanko et al. (2006), se a empresa aumenta o preço e tem uma pequena queda nas vendas, pode-se supor que a receita de vendas aumentará, já que o aumento do preço compensará a queda na quantidade. Mas, se com o aumento do preço, as vendas caírem drasticamente, pressupõe-se que a receita de vendas diminuirá também.

Isso vai depender da elasticidade-preço da demanda, que impacta consideravelmente a previsão da receita total: para um produto com demanda elástica, a receita total aumenta em função de uma redução no preço; mas para um produto com demanda inelástica, a receita total aumenta em função de um aumento no preço. (CHURCHIL, 2000)

Gold e Pray (1984) pesquisaram, em diversos simuladores empresariais, esta elasticidade-preço da demanda, que apresentou-se sempre negativa, indicando que um aumento no preço implica em redução na quantidade vendida.

Lemos (2011) também pesquisou esta elasticidade, mas em uma empresa simulada participante de um Jogo de Empresas específico. Ao estudar como as decisões de preço 
podem influenciar a receita de uma empresa, a autora concluiu que nem sempre uma redução de preços garante o aumento da receita e vice-versa e, consequentemente que, na prática, os conceitos microeconômicos não se comportam necessariamente da forma como a teoria apresenta.

Honaiser e Sauaia (2008), novamente no contexto de Jogos de Empresas, pesquisaram a influência do preço na demanda, mas considerando o preço relativo de cada empresa virtual em comparação ao preço médio da indústria.

Já os resultados obtidos por Oliveira e Alves (2012), também no ambiente de um Jogo de Empresas, confirmam a literatura, apontando a política de preços como instrumento útil e válido no apoio a estratégias de Marketing. Os autores analisaram a relação entre diferentes políticas de preços e os resultados obtidos com essas políticas pelas empresas participantes do jogo, concluindo - especificamente no ambiente testado - que a política de maximização da participação no mercado foi a que teve o potencial de gerar o maior faturamento.

Mas tal elasticidade depende de vários fatores, inclusive da indústria em questão. Na área de saúde, Pires (2010) verificou que o aumento do preço por cuidados médicos levou a uma redução mais do que proporcional das quantidades procuradas, ocasionando uma diminuição na receita.

\section{METODOLOGIA}

A ideia consistiu em estabelecer um "Laboratório de Logística", uma estrutura de simulação de ambiente empresarial nos moldes do "Laboratório de Gestão" (SAUAIA, 2007, 2010), mas cujo motor foi o BR-LOG (BOUZADA; SALIBY, 2001), simulador contextualizado em um oligopólio onde empresas fabricam e tentam vender produtos fictícios, que diferem entre si em termos de magnitude e sazonalidade da demanda, preço, margem de lucro potencial e complexidade de produção, armazenagem e distribuição.

O jogo foi aplicado, durante os anos de 2011, 2012, 2013 e 2014 em:

$\checkmark$ quatro turmas de Especialização em Logística do COPPEAD/UFRJ, constituindo a própria disciplina, Jogo de Logística;

$\checkmark \quad$ três turmas de graduação em Administração da Escola Superior de Propaganda e Marketing (ESPM), nas suas três filiais (Rio de Janeiro, São Paulo e Porto Alegre), como parte integrante e como uma das formas de avaliação de disciplinas relacionadas a Logística Empresarial; e

$\checkmark \quad$ em quatro turmas do CBA em Logística (pós-gradução) do Instituto Brasileiro de Mercado de Capitias (IBMEC/RJ), constituindo a própria disciplina, Jogo de Logística.

Cabe deixar claro que o objetivo deste trabalho não consistiu em testar o simulador em ambiente de sala de aula, mas sim em usá-lo para testar elementos de pesquisa, conforme destacado na Introdução.

Também não se pretendeu, neste momento, que os participantes do jogo conduzissem pesquisa aplicada. Tal tipo de atividade é importante dentro do conceito mais amplo do Laboratório de Gestão (SAUAIA, 2010), mas pareceu mais oportuno considerá-la apenas em termos de oportunidades futuras.

Nas turmas da ESPM e do COPPEAD, a aplicação se deu de forma remota, com os participantes enviando suas decisões semanalmente por email para o aplicador, que rodou o simulador e devolveu os relatórios para as equipes, também semanalmente e por email. Os participantes precisaram usar algo em torno de 2 horas de computador semanais para tomar as decisões.

Nas turmas do IBMEC, a aplicação foi presencial, com o aplicador estando junto à turma durante as 15 horas divididas nas 5 aulas dedicadas à disciplina 
Em todas as turmas, cada equipe fez uma apresentação, após o final do jogo, destacando a estratégia da empresa, como as tarefas foram divididas, os principais erros e acertos da equipe, as ferramentas de apoio utilizadas e qual foi o aprendizado resultante da atividade. Nesta apresentação, todos os integrantes de cada equipe tiveram que se manifestar oralmente.

Ao final das apresentações, o aplicador atribuiu uma nota individual para cada aluno, tentando refletir o seu nível de participação na atividade, a coerência das suas decisões e a intensidade do seu empenho.

Cada equipe também recebeu uma nota coletiva, refletindo simplesmente o desempenho financeiro da empresa administrada por ela.

A nota final de cada aluno na atividade foi obtida pelo cálculo da média aritmética entre a sua nota individual na apresentação (subjetiva) e a nota coletiva (objetiva).

Estes procedimentos encerraram a coleta de dados. Os mesmos foram tratados para atender os objetivos de pesquisa, conforme detalhado a seguir.

Para testar a relação dos atributos relacionados à venda dos produtos (nível de serviço logístico e preço) com os indicadores financeiros das empresas no jogo (receita, custo total, lucro e retorno sobre o investimento), foram realizadas algumas análises de correlação linear envolvendo seis variáveis e, considerando, indistintamente, as empresas de todas as turmas que participaram do jogo. Os valores das seis variáveis envolvidas na análise foram coletados, para cada empresa, conforme especificado a seguir:

1. nível de serviço logístico (\%) - percentual das unidades encomendadas que foi efetivamente entregue, considerando todos os atacadistas e todos os produtos;

2. preço $(\%)$ - média, para todos os produtos, do preço médio (para todas as semanas) praticado pela empresa em cada produto, representado como um percentual do preço médio geral (para todas as empresas) de cada produto

3. receita $(\mathrm{R} \$)$ - total vendido pela empresa ao longo de todas as semanas, em termos de volume financeiro e considerando todos os produtos e atacadistas;

4. custo total $(\mathrm{R} \$)$ - custo total da empresa ao longo de todas as semanas;

5. resultado final $(\mathrm{R} \$)$ - receita acumulada menos custo total acumulado ao longo de todas as semanas;

6. retorno sobre o investimento (\%) - resultado final dividido pelo valor financeiro investido em ativos.

Procurou-se, inicialmente, examinar a correlação linear entre o nível de serviço logístico das empresas e (i) sua receita (hipótese 1a, definida na seção 1.2), (ii) seu custo total (hipótese 1b); (iii) seu resultado final (1c) e (iv) seu retorno sobre o investimento (1d). Em seguida, atacando um problema de pesquisa similar ao estudado por Lemos (2011) e Oliveira e Alves (2012) - a elasticidade-preço da demanda - foi calculada a correlação linear entre o preço das empresas e (i) sua receita (hipótese 2a) e (ii) seu resultado final (2b). Finalmente, uma análise de correlação linear entre o nível de serviço logístico e o preço das empresas permitiu verificar a ocorrência ou não de coerência estratégica (empresas com melhor nível de serviço logístico praticando preços mais altos) (hipótese 3).

Os resultados dos testes foram comparados às conclusões teóricas e empíricas (mas não experimentais!) encontradas na literatura acerca dos elementos envolvidos na análise e da sua influência no mundo real, assim como fizeram Lima e Sauaia (2008) em relação ao impacto do investimento em P\&D nos resultados empresariais, Ribeiro (2012) quanto ao impacto de um bom planejamento da produção nos custos de produção, Lemos (2011) e Oliveira e Alves (2012) em relação ao impacto do preço na receita de vendas e no desempenho financeiro, Silva e Sauaia (2012) quanto ao impacto do cumprimento do Plano de Marketing na redução das incertezas e na melhoria do desempenho e Rivera, Domenico e 
Sauaia (2014) em relação à influência da heterogeneidade dos Times de Alta Gerência - em termos de valores individuais - no seu desempenho.

Por exemplo, de acordo com a teoria, espera-se, em princípio, que as empresas com melhor nível de serviço logístico o consigam encerrando custos totais mais elevados (hipótese de pesquisa 2b) mas, em compensação, obtendo receitas de vendas mais expressivas (hipótese 2a).

\section{RESULTADOS}

Os valores das seis variáveis descritas na seção anterior foram coletados para as 41 empresas que participaram das 11 turmas mencionadas anteriormente e estão apresentados na tabela 1 a seguir.

Tabela 1 - Nível de serviço logístico, preço, receita, custo total, resultado final e retorno sobre o investimento das 41 empresas administradas pelos jogadores das 11 turmas

\begin{tabular}{|c|c|c|c|c|c|c|c|c|c|c|c|}
\hline Instituição & Turma & Empresa & \begin{tabular}{|c|} 
Nível de \\
serviço \\
logístico
\end{tabular} & Preço & & Receita & & sto total & & Itado final & $\begin{array}{c}\text { Retorno } \\
\text { sobre o } \\
\text { investimento }\end{array}$ \\
\hline \multirow{16}{*}{ C } & \multirow{4}{*}{1} & 1 & $37 \%$ & $100 \%$ & $\mathrm{R} \$$ & 3.006 .693 & $\mathrm{R} \$$ & 6.581 .125 & $-\mathrm{R} \$$ & 3.574 .432 & $-248 \%$ \\
\hline & & 2 & $88 \%$ & $98 \%$ & $\mathrm{R} \$$ & $\begin{array}{r}6.296 .653 \\
\end{array}$ & $\mathrm{R} \$$ & 10.352 .123 & $-\mathrm{R} \$$ & 4.055 .471 & $-115 \%$ \\
\hline & & 3 & $74 \%$ & $104 \%$ & $\mathrm{R} \$$ & 11.162 .083 & $\mathrm{R} \$$ & $\begin{array}{l}6.711 .458 \\
\end{array}$ & $\mathrm{R} \$$ & 4.450 .626 & $226 \%$ \\
\hline & & 4 & $82 \%$ & $98 \%$ & $\mathrm{R} \$$ & 3.078 .102 & $\mathrm{R} \$$ & 9.155 .559 & $-\mathrm{R} \$$ & 6.077 .457 & $-410 \%$ \\
\hline & \multirow{4}{*}{2} & 1 & $71 \%$ & $104 \%$ & $\mathrm{RS}$ & 27.001 .240 & $\mathrm{R} \$$ & 8.840 .718 & $\mathrm{R} \$$ & 18.160 .522 & $1808 \%$ \\
\hline & & 2 & $37 \%$ & $89 \%$ & $\mathrm{R} \$$ & 25.649 .144 & $\mathrm{R} \$$ & 6.506 .229 & $\mathrm{R} \$$ & $\begin{array}{l}19.142 .915 \\
\end{array}$ & $2223 \%$ \\
\hline & & 3 & $34 \%$ & $96 \%$ & $\mathrm{R} \$$ & 6.098 .585 & $\mathrm{R} \$$ & 3.120 .693 & $\mathrm{R} \$$ & 2.977 .892 & $337 \%$ \\
\hline & & 4 & $52 \%$ & $112 \%$ & $\mathrm{R} \$$ & 26.616 .431 & $\mathrm{R} \$$ & 7.312 .530 & $\mathrm{R} \$$ & 19.303 .901 & $1384 \%$ \\
\hline & \multirow{4}{*}{3} & 1 & $82 \%$ & $94 \%$ & $\mathrm{R} \$$ & 22.172 .663 & $\mathrm{R} \$$ & $\begin{array}{l}14.126 .666 \\
\end{array}$ & $\mathrm{R} \$$ & 8.045 .997 & $344 \%$ \\
\hline & & 2 & $41 \%$ & $99 \%$ & $\mathrm{R} \$$ & 13.110 .500 & $\mathrm{R} \$$ & $\begin{array}{l}8.845 .972 \\
\end{array}$ & $\mathrm{R} \$$ & 4.264 .528 & $244 \%$ \\
\hline & & 3 & $51 \%$ & $113 \%$ & $\mathrm{R} \$$ & $\begin{array}{l}43.469 .037 \\
\end{array}$ & $\mathrm{R} \$$ & 10.090 .283 & $\mathrm{R} \$$ & $\begin{array}{l}33.378 .753 \\
\end{array}$ & $2427 \%$ \\
\hline & & 4 & $70 \%$ & $94 \%$ & $\mathrm{R} \$$ & 12.087 .743 & $\mathrm{R} \$$ & 6.500 .947 & $\mathrm{R} \$$ & 5.586 .796 & $260 \%$ \\
\hline & \multirow{4}{*}{4} & 1 & $56 \%$ & $96 \%$ & $\mathrm{R} \$$ & $\begin{array}{l}7.685 .173 \\
\end{array}$ & $\mathrm{R} \$$ & 11.105 .413 & $-\mathrm{R} \$$ & 3.420 .240 & $-92 \%$ \\
\hline & & 2 & $53 \%$ & $92 \%$ & $\mathrm{R} \$$ & 39.825 .213 & $\mathrm{R} \$$ & $\begin{array}{l}7.393 .407 \\
\end{array}$ & $\mathrm{R} \$$ & 32.431 .806 & $3213 \%$ \\
\hline & & 3 & $32 \%$ & $91 \%$ & $\mathrm{R} \$$ & 4.839 .207 & $\mathrm{R} \$$ & 3.171 .450 & $\mathrm{R} \$$ & 1.667 .756 & $270 \%$ \\
\hline & & 4 & $91 \%$ & $121 \%$ & $\mathrm{RS}$ & 40.054 .089 & $\mathrm{R} \$$ & 13.160 .684 & $\mathrm{R} \$$ & 26.893 .405 & $1751 \%$ \\
\hline \multirow{11}{*}{$\begin{array}{l}\mathbf{E} \\
\mathbf{S} \\
\mathbf{P} \\
\mathbf{M}\end{array}$} & \multirow{4}{*}{1} & 1 & $43 \%$ & $103 \%$ & $\mathrm{R} \$$ & 15.446 .823 & $\mathrm{RS}$ & $\begin{array}{r}.971 .499 \\
\end{array}$ & $\mathrm{RS}$ & 11.475 .324 & $965 \%$ \\
\hline & & 2 & $33 \%$ & $107 \%$ & $\mathrm{R} \$$ & $\begin{array}{l}4.262 .496 \\
\end{array}$ & $\mathrm{R} \$$ & 2.244 .889 & $\mathrm{R} \$$ & $\begin{array}{l}2.017 .607 \\
\end{array}$ & $280 \%$ \\
\hline & & 3 & $27 \%$ & $95 \%$ & $\mathrm{R} \$$ & 2.209 .213 & $\mathrm{R} \$$ & 3.404 .091 & $-\mathrm{R} \$$ & 1.194 .878 & $-70 \%$ \\
\hline & & 4 & $3 \%$ & $95 \%$ & $\mathrm{R} \$$ & 267.250 & $\mathrm{R} \$$ & 1.203 .740 & $-\mathrm{R} \$$ & 936.490 & $-236 \%$ \\
\hline & \multirow{3}{*}{2} & 1 & $0 \%$ & $99 \%$ & $\mathrm{R} \$$ & 62.608 & $\mathrm{R} \$$ & 1.047 .135 & $-\mathrm{R} \$$ & 984.527 & $-339 \%$ \\
\hline & & 2 & $20 \%$ & $102 \%$ & $\mathrm{R} \$$ & 2.013 .538 & $\mathrm{R} \$$ & 4.255 .534 & $-\mathrm{R} \$$ & 2.241 .996 & $-479 \%$ \\
\hline & & 3 & $5 \%$ & $99 \%$ & $\mathrm{R} \$$ & 312.394 & $\mathrm{R} \$$ & 1.741 .724 & $-\mathrm{R} \$$ & 1.429 .330 & $-318 \%$ \\
\hline & \multirow{4}{*}{3} & 1 & $9 \%$ & $99 \%$ & $\mathrm{R} \$$ & 4.901.139 & $\mathrm{R} \$$ & 3.157 .868 & $\mathrm{R} \$$ & 1.743 .272 & $173 \%$ \\
\hline & & 2 & $1 \%$ & $101 \%$ & $\mathrm{R} \$$ & $\begin{array}{l}157.830 \\
\end{array}$ & $\mathrm{R} \$$ & 3.932 .675 & $-\mathrm{R} \$$ & 3.774 .845 & $-309 \%$ \\
\hline & & 3 & $2 \%$ & $100 \%$ & $\mathrm{R} \$$ & 894.444 & $\mathrm{R} \$$ & 4.723 .507 & $-\mathrm{R} \$$ & 3.829 .063 & $-122 \%$ \\
\hline & & 4 & $9 \%$ & $100 \%$ & $\mathrm{R} \$$ & 370.004 & $\mathrm{R} \$$ & 3.454 .359 & $-\mathrm{R} \$$ & 3.084 .355 & $-395 \%$ \\
\hline \multirow{14}{*}{$\begin{array}{l}\text { I } \\
\text { B } \\
\text { M } \\
\text { E } \\
\text { C }\end{array}$} & \multirow{4}{*}{1} & 1 & $43 \%$ & $98 \%$ & $\mathrm{R} \$$ & 6.547 .488 & $\mathrm{R} \$$ & 3.703 .345 & $\mathrm{R} \$$ & 2.844 .143 & $454 \%$ \\
\hline & & 2 & $67 \%$ & $103 \%$ & $\mathrm{R} \$$ & 7.747 .723 & $\mathrm{R} \$$ & 7.409 .757 & $\mathrm{R} \$$ & 337.966 & $11 \%$ \\
\hline & & 3 & $76 \%$ & $102 \%$ & $\mathrm{R} \$$ & 8.207 .995 & $\mathrm{R} \$$ & 6.028 .372 & $\mathrm{R} \$$ & 2.179 .622 & $213 \%$ \\
\hline & & 4 & $31 \%$ & $97 \%$ & $\mathrm{RS}$ & 2.338 .251 & $\mathrm{RS}$ & 5.464 .900 & $-\mathrm{R} \$$ & 3.126 .649 & $-131 \%$ \\
\hline & \multirow{3}{*}{2} & 1 & $25 \%$ & $99 \%$ & $\mathrm{R} \$$ & 1.737 .581 & $\mathrm{R} \$$ & 1.137 .071 & $\mathrm{R} \$$ & 600.510 & $164 \%$ \\
\hline & & 2 & $27 \%$ & $111 \%$ & $\mathrm{R} \$$ & 4.015 .104 & $\mathrm{R} \$$ & 1.531 .360 & $\mathrm{R} \$$ & 2.483 .744 & $685 \%$ \\
\hline & & 3 & $66 \%$ & $93 \%$ & $\mathrm{R} \$$ & 10.737 .292 & $\mathrm{R} \$$ & 8.536 .197 & $\mathrm{R} \$$ & 2.201 .095 & $269 \%$ \\
\hline & \multirow{4}{*}{3} & 1 & $28 \%$ & $103 \%$ & $\mathrm{R} \$$ & $\begin{array}{l}3.504 .941 \\
\end{array}$ & $\mathrm{R} \$$ & 5.520 .352 & $-\mathrm{R} \$$ & 2.015 .411 & $-123 \%$ \\
\hline & & 2 & $28 \%$ & $102 \%$ & $\mathrm{R} \$$ & 4.165 .980 & $\mathrm{R} \$$ & 1.997 .778 & $\mathrm{R} \$$ & 2.168 .202 & $417 \%$ \\
\hline & & 3 & $47 \%$ & $97 \%$ & $\mathrm{R} \$$ & 7.287.151 & $\mathrm{R} \$$ & 2.464 .224 & $\mathrm{R} \$$ & 4.822 .927 & $657 \%$ \\
\hline & & 4 & $68 \%$ & $99 \%$ & $\mathrm{R} \$$ & 6.209 .964 & $\mathrm{R} \$$ & 4.550 .230 & $\mathrm{R} \$$ & 1.659 .734 & $304 \%$ \\
\hline & \multirow{3}{*}{4} & 1 & $56 \%$ & $92 \%$ & $\mathrm{R} \$$ & 3.644 .397 & $\mathrm{R} \$$ & 4.078 .705 & -R\$ & 434.308 & $-59 \%$ \\
\hline & & 2 & $81 \%$ & $114 \%$ & $\mathrm{RS}$ & 18.688 .732 & $\mathrm{R} \$$ & 13.451 .286 & $\mathrm{R} \$$ & 5.237 .446 & $363 \%$ \\
\hline & & 3 & $44 \%$ & $104 \%$ & $\mathrm{RS}$ & $\begin{array}{l}5.969 .097 \\
\end{array}$ & $\mathrm{R} \$$ & 2.342 .800 & $\mathrm{RS}$ & 3.626 .297 & $994 \%$ \\
\hline
\end{tabular}

Fonte: elaboração própria

De posse desses dados, foi possível realizar as análises de correlação anteriormente mencionadas e testar as hipóteses estabelecidas na seção 1.2.

\subsection{RELAÇÃO ENTRE O NÍVEL DE SERVIÇO LOGÍSTICO E A RECEITA DAS EMPRESAS}

O coeficiente de correlação linear de Pearson $(r)$ obtido foi de 0,51 , indicando uma correlação positiva moderada (entre 0,3 e 0,6 ). Este valor é estatisticamente diferente de zero (ausência de correlação), a 1\% de significância.

Em outras palavras, é possível afirmar que, de uma maneira geral e com moderada convicção, as empresas que conseguiram um melhor nível de serviço logístico também 
conseguiram maiores receitas, o que contribui para a confirmação parcial da hipótese 1a - o nível de serviço é capaz de impactar positivamente a receita das empresas.

\subsection{RELAÇÃO ENTRE O NÍVEL DE SERVIÇO LOGÍSTICO E O CUSTO TOTAL DAS EMPRESAS}

Neste caso, houve um alinhamento também positivo, mas certamente mais acentuado do que na relação anterior. $\mathrm{O}$ valor de 0,76 obtido para $r$ revela uma correlação positiva forte (acima de 0,6). Este valor é estatisticamente diferente de zero, a 1\% de significância.

Assim, é possível afirmar que, de uma maneira geral e com forte convicção, as empresas que conseguiram um melhor nível de serviço logístico também tiveram maiores custos totais, o que contribui para a confirmação plena da hipótese $1 \mathrm{~b}$ - o nível de serviço é capaz de impactar positivamente o custo total das empresas.

\subsection{RELAÇÃO ENTRE O NÍVEL DE SERVIÇO LOGÍSTICO E O RESULTADO FINAL DAS EMPRESAS}

Já para esta situação, há uma relação mais nebulosa entre as variáveis. O coeficiente $r$ apresentou valor de 0,33 , o que caracteriza uma correlação positiva e moderada. Este valor é estatisticamente diferente de zero, a 5\% de significância.

Desta forma, é possível afirmar com moderada convicção que as empresas que conseguiram um melhor nível de serviço logístico também obtiveram resultados finais superiores, o que contribui para a confirmação parcial da hipótese 1c - o nível de serviço é capaz de impactar positivamente o resultado final das empresas.

\subsection{RELAÇÃO ENTRE O NÍVEL DE SERVIÇO LOGÍSTICO E O RETORNO SOBRE INVESTIMENTO DAS EMPRESAS}

A relação, nesta situação, é parecida com a da relação anterior. O valor obtido para $r$ foi de 0,30, indicando novamente uma correlação positiva moderada. Este valor é estatisticamente diferente de zero, a 10\% de significância.

Por conseguinte, é possível afirmar com moderada convicção que as empresas que conseguiram um melhor nível de serviço logístico também obtiveram melhores retornos sobre o investimento, o que contribui para a confirmação parcial da hipótese $1 \mathrm{~d}$ - o nível de serviço é capaz de impactar positivamente o retorno sobre o investimento das empresas.

\subsection{RELAÇÃO ENTRE O PREÇO E A RECEITA DAS EMPRESAS}

$\mathrm{O}$ alinhamento foi ligeiramente mais acentuado do que nos casos anteriores. O valor obtido para $r$ foi de 0,35 , sugerindo uma correlação positiva moderada. Este valor é estatisticamente diferente de zero, a 5\% de significância.

Ou seja, é possível afirmar que, de uma maneira geral e com moderada convicção, as empresas que trabalharam com preços mais altos também alcançaram maiores receitas, o que contribui para a confirmação parcial da hipótese $2 \mathrm{a}$ - o preço é capaz de impactar a receita das empresas.

\subsection{RELAÇÃO ENTRE O PREÇO E O RESULTADO FINAL DAS EMPRESAS}

$\mathrm{O}$ alinhamento foi parecido com o da última relação. $\mathrm{O}$ valor de $r$ de 0,33 implica em uma correlação positiva moderada. Este valor é estatisticamente diferente de zero, a 5\% de significância.

É possível afirmar que, de uma maneira geral e com moderada convicção, as empresas que trabalharam com preços mais altos também conseguiram resultados finais melhores, o que contribui para a confirmação parcial da hipótese $2 \mathrm{~b}$ - o preço é capaz de impactar o resultado final das empresas. 


\subsection{RELAÇÃO ENTRE O NÍVEL DE SER VIÇO LOGÍSTICO E O PREÇO DAS EMPRESAS}

Ocorreu uma quase completa ausência de relação entre as variáveis. O coeficiente $r$ obtido é bem baixo $(0,19)$, revelando uma ausência de correlação (abaixo de 0,3). Este valor não é estatisticamente diferente de zero, a $10 \%$ de significância.

Fica claro, assim, que não é possível afirmar que as empresas que conseguiram um melhor nível de serviço logístico também praticaram preços mais altos, o que contribui para a rejeição da hipótese 3 - o nível de serviço e o preço de venda praticado pelas empresas caminham na mesma direção.

\section{CONSIDERAÇÕES FINAIS}

Este trabalho procurou fazer uso de uma metodologia tipicamente de ensino (os Jogos de Empresas) para servir de palco experimental para pesquisa, em uma tentativa de verificar ou refutar teoria na área de Logística.

As variáveis nível de serviço, preço, receita, custo total, resultado final e retorno sobre o investimento de empresas virtuais participantes de onze aplicações de um jogo de Logística em turmas de graduação e pós-graduação foram utilizados como dados experimentais para testar, na forma de hipóteses de pesquisa, algumas relações entre estas variáveis, que a teoria prega existir.

A hipótese 1a - o nível de serviço é capaz de impactar positivamente a receita das empresas - foi aceita parcialmente. Este resultado empírico vai ao encontro - parcialmente do que diz Ballou (1993) sobre a possibilidade de um bom desempenho em nível de serviço gerar valor e agradar os clientes - o que também sugerem Gomes e Ribeiro (2004) - e aumentar as vendas. Segundo o primeiro autor, quanto maior o nível de serviço, maiores serão as receitas, em geral, o que, a propósito, também sugere o bom senso.

A hipótese 1 b - o nível de serviço é capaz de impactar positivamente o custo total das empresas - também foi confirmada, mas plenamente, o que está de acordo com Bowersox e Closs (1996), que dizem que o serviço perfeito seria custoso, com Ballou (1993), que afirma um maior nível de serviço logístico custa mais que níveis menores, e com Porter (1980), que indica ser normalmente custoso oferecer um nível de serviço bem superior à concorrência. Tais colocações teóricas, assim como no caso anterior, também corroboram o senso comum.

Já as hipóteses 1c e 1d - o nível de serviço é capaz de impactar positivamente o resultado final e o retorno sobre o investimento das empresas, respectivamente - foram aceitas apenas de forma parcial (e com menos convicção do que a hipótese $1^{\mathrm{a}}$ ).

A não confirmação plena destas hipóteses está parcialmente em linha com a teoria pregada por Ballou (1993), que lembra que o maior lucro não é necessariamente atingido com maiores níveis de serviço. Essa ideia é justificada e complementada por Bowersox e Closs (1996), que sugerem uma permanente indagação do gestor logístico acerca da viabilidade do custo associado a conseguir prestar serviço a um nível específico. Gomes e Ribeiro (2004) também colocam que este gestor logístico precisa se preocupar com a eficiência em termos de custos no momento de almejar determinados níveis de serviço.

Se as hipóteses fossem confirmadas de forma plena ou se a teoria indicasse o contrário do que indica, constituir-se-ia uma sugestão implícita de existência de uma estratégia vencedora, de maximização do nível de serviço. E, se assim fosse, todas as empresas tentariam praticar essa estratégia, elevando ao máximo o nível de serviço. E isso, obviamente, não é o que ocorre na realidade.

Foram confirmadas também parcialmente ambas as hipóteses relacionadas ao preço, 2a e 2 b - o preço é capaz de impactar a receita e o resultado final das empresas, respectivamente - o que refuta, parcialmente, a teoria, que não é consensual a esse respeito. 
Besanko et al. (2006) e Churchil (2000) sugerem que o aumento dos preços pode acarretar uma diminuição ou aumento da receita, variando se a quantidade vendida diminuir muito ou pouco, o que vai depender da elasticidade-preço da demanda, o que também foi confirmado experimentalmente em um Jogo de Empresas por Lemos (2011).

Já Oliveira e Alves (2012) - também em um Jogo de Empresas - e Pires (2010) encontraram resultados mais incisivos sobre o impacto do preço na receita e no resultado total, verificando que uma redução (aumento) no preço gerou aumento (diminuição) na receita, resultado contrário ao encontrado pela pesquisa apresentada no presente artigo.

Em vista dessas divergências, parece interessante investigar, oportunamente, em que indústrias/contextos o preço é capaz de impactar positiva ou negativamente ou ainda não impactar a receita e o resultado final das empresas.

A última hipótese, que relaciona os dois principais construtos desta pesquisa - o nível de serviço e o preço de venda praticado pelas empresas caminham na mesma direção foi rejeitada com veemência.

Esse resultado vai totalmente de encontro às recomendações de Kotler e Armstrong (2000), para os quais é preciso sincronizar a estratégia de apreçamento com as estratégias do marketing mix (que incluem o nível de serviço) e de Porter (1980), que sugere que a consequência natural do custo de oferecer um nível de serviço superior é a necessidade de praticar um preço premium, capaz de compensar o custo mais elevado.

Tal discordância, nesse caso, não caracteriza que os resultados experimentais estão refutando os teóricos; apenas indica que os participantes do jogo não estão, de maneira geral, atendendo as sugestões da teoria. A real motivação para este descolamento ter acontecido por ser objeto de uma futura pesquisa qualitativa.

Naturalmente, os resultados deste trabalho precisam ser vistos com as devidas ressalvas, já que um jogo de empresas depende de um simulador, que: (i) se aplica a um contexto específico de produtos, matérias-primas e dinâmicas de concorrência, podendo, eventualmente, ter pouca representatividade no contexto analisado; e (ii) é construído em cima de modelo que, por sua vez, nunca deixa de ser uma simplificação da realidade, em função de não contemplar - tanto por conveniência do algoritmo quanto por questões de parcimônia - muitas variáveis relevantes.

Além disso, as empresas participantes das aplicações do jogo foram geridas por alunos (com maior ou menor grau de experiência na área), muitos deles de graduação, e não por profissionais extremamente experientes na área de Logística. Desta forma, seria muito leviano afirmar que os resultados seriam exatamente os mesmos se as decisões que impactam os indicadores estudados tivessem sido tomadas por profissionais de mercado pertencentes a empresas reais, o que caracterizaria uma pesquisa empírica, e não experimental, como foi a relatada neste artigo.

Não obstante, os resultados experimentais aqui apresentados podem e devem ser levados em conta, pois lançam uma luz a respeito de alguns modelos teóricos, ora verificando-os, ora refutando-os, contribuindo para um melhor entendimento dos mesmos, mas nunca tendo a pretensão de serem definitivos acerca de tais fenômenos.

Por exemplo, o impacto positivo do nível de serviço nos custos da empresa parece ser mais verdadeiro (correlação forte), à luz dos resultados desta pesquisa, do que o impacto na receita da empresa (correlação moderada); e mais verdadeiro ainda do que o impacto no resultado total e no retorno sobre o investimento (correlações também moderadas, mas mais fracas).

Tais posições relativas das correlações e o sumário dos resultados dos testes das hipóteses de pesquisa podem ser mais bem visualizadas na figura 1 a seguir.

Como ideias para estudos futuros, sugere-se, conforme já citado, investigar o impacto do preço na receita e no resultado das empresas em diferentes contextos, e pesquisar 
qualitativamente o motivo das empresas virtuais não estarem alinhando nível de serviço e preço, como recomenda a teoria.

Figura 1 - Resultado das hipóteses de pesquisa

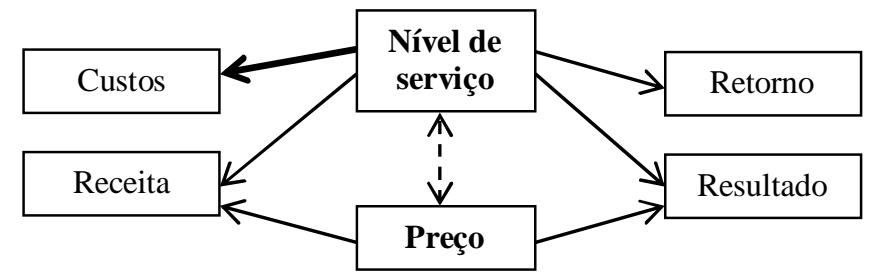

$\longrightarrow$ Relação forte $\longrightarrow$ Relação moderada $\quad-\rightarrow$ Relação fraca/inexistente

Fonte: elaboração própria

Outra ideia consiste em dar continuidade ao Laboratório de Logística para testar experimentalmente mais alguns elementos de pesquisa na área de Logística, conforme sugeridos por Bouzada (2010).

\section{REFERÊNCIAS BIBLIOGRÁFICAS}

[1] AZEREDO, S.; ORNELLAS, A.; RAMOS, R. Jogos de empresas aplicados à logística empresarial: um panorama dos modelos disponíveis no país. In: ENCONTRO NACIONAL DE ENGENHARIA DE PRODUÇÃO, 26., 2006, Fortaleza. Anais... Fortaleza: ENEGEP, 2006.

[2] BALLOU, R. Logística Empresarial. São Paulo: Atlas, 1993.

[3] BESANKO, D.; DRANOVE, D.; SHANLEY, M.; SHAEFER, S. A Economia da Estratégia. Porto Alegre: Bookman, 2006.

[4] BONOCIELLI Jr., S.; LOPES, P. Estudo e Modelagem do Mercado de Capitais em Jogo de Empresas Geral. In: SEMINÁRIOS EM ADMINISTRAÇÃO, 11., 2008, São Paulo. Anais... São Paulo: SEMEAD, 2008.

[5] BOUZADA, M. Um jogo de Logística genuinamente brasileiro. Dissertação (Mestrado em Administração) - Instituto de Pós-Graduação e Pesquisa em Administração. Rio de Janeiro: UFRJ/COPPEAD, 2001.

[6] BOUZADA, M. Laboratório de logística: uma proposta de metodologia de pesquisa. In: SIMPÓSIO DE ADMINISTRAÇÃ̃O DA PRODUÇÃO, LOGÍSTICA E OPERAÇÕES INTERNACIONAIS, 13., 2010, São Paulo. Anais... São Paulo: SIMPOI, 2010.

[7] BOUZADA, M. (Org.). Jogando Logística no Brasil. Curitiba: CRV, 2011.

[8] BOUZADA, M.; SALIBY, E. Um Jogo de Logística genuinamente brasileiro. In: SIMPÓSIO DE PESQUISA OPERACIONAL E LOGÍSTICA DA MARINHA, 4., 2001, Rio de Janeiro. Anais... Rio de Janeiro: SPOLM, 2001.

[9] BOWERSOX, D., CLOSS, D. Logistical management: the integrated supply chain process. New York: McGraw-Hill, 1996.

[10] CEL. Centro de Estudos em Logística do COPPEAD/UFRJ. Jogos de Empresas. Disponível em: <http://www.centrodelogistica.org/new/fs-jogos.htm>. Acesso em: 30 jun. 08

[11] CHURCHIL, G. Marketing: criando valor para o cliente. São Paulo: Saraiva, 2000.

[12] DAVIS, J.; EISENHARDT, K.; BINGHAM, C. Developing theory through simulation methods. Academy of Management Review, v. 32, n. 2, p. 480-499, 2007. 
[13] GOLD, S.; PRAY, T. Modeling non-price factors in the demand functions of computerized business simulations. In: ASSOCIATION FOR BUSINESS SIMULATION AND EXPERIENTIAL LEARNING CONFERENCE, 11., 1994, Stillwater (OK, USA). Proceedings... Stillwater: ABSEL, 1994.

[14] GOMES, C.; RIBEIRO, P. Gestão da Cadeia de Suprimentos Integrada à Tecnologia da Informação. São Paulo: Pioneira Thomson Learning, 2004.

[15] HESKETT, J. Controlling customer logistics service. International Journal of Physical Distribution, p. 141-145, Jun 1971.

[16] HONAISER, E.; SAUAIA, A. Desenvolvimento e Aplicação de um Modelo para Previsão de Demanda em Jogos de Empresas. RAC-Eletrônica, Curitiba, v. 2, n. 3, p. 470-485, Set./Dez. 2008.

[17] KOTLER, P.; ARMSTRONG, G. Introdução ao Marketing. Rio de Janeiro: LTC, 2000.

[18] LEMOS, M. Decisões de preço em jogos de empresas: o estudo das elasticidades e do ponto de equilíbrio como ferramentas de apoio à decisão. Revista LAGOS - UFF (Volta Redonda), v. 1, n. 2, p. 1-16, 2011.

[19] LIMA, M. Jogos de empresa e operações logísticas. Artigos CEL, 2004. Disponível em: <http://www.cel.coppead.ufrj.br/fr-art-jogos_empresa.htm>. Acesso em: 30 jun. 08.

[20] LIMA, M.; SAUAIA, A. Impacto dos Investimentos em P\&D nos Resultados Empresariais: um Estudo Laboratorial com Jogos de Empresas. In: SIMPÓSIO DE GESTÃO DA INOVAÇÃO TECNOLÓGICA, 25., 2008, Brasília. Anais... Brasília: ANPAD, 2008.

[21] OLIVEIRA, M.; ALVES, C. Política de Preços no Desempenho de Empresas: um Estudo com Simulador Organizacional de Estratégia. Sociedade, Contabilidade $\boldsymbol{e}$ Gestão, v. 7, n. 1, p. 140-155, 2012.

[22] PIRES, B. O impacto da variação do preço na procura de cuidados de saúde. Dissertação (Mestrado em Gestão da Saúde) - Escola Nacional de Saúde Pública. Lisboa: Universidade Nova de Lisboa, 2010.

[23] PORTER, M. Competitive strategy. New York: The Free Press, 1980.

[24] RIBEIRO, R. Planejamento da produção para atender a demanda com minimização de custos em um jogo de empresas. In: SEMINÁRIOS DE ADMINISTRAÇÃO DA USP, 15., 2012, São Paulo. Anais... São Paulo: SEMEAD, 2012.

[25] RIVERA, J.; DOMENICO, S.; SAUAIA, A. Influência da Dissimilaridade de Valores Individuais no Resultado de Times de Alta Gerência: um estudo em laboratório de gestão. Revista Brasileira de Gestão de Negócios, São Paulo, v. 16, n. 50, p. 60-74, jan./mar. 2014.

[26] SAUAIA, A. Workshop em Jogos de Empresas: uma vivência para coordenadores, docentes e pesquisadores. In: ENCONTRO DE ENSINO E PESQUISA EM ADMINISTRAÇÃO E CONTABILIDADE, 1., 2007, Recife. Anais... Recife: ANPAD, 2007.

[27] SAUAIA, A. Laboratório de gestão: simulador organizacional, jogo de empresas e pesquisa aplicada. 2 ed. Barueri: Manole, 2010.

[28] SILVA, S.; SAUAIA, A. Avaliação do Previsto X Realizado num Jogo de Empresas. In: CONGRESSO DE ADMINISTRAÇÃO, SOCIEDADE E INOVAÇÃO, 1., 2012, Volta Redonda (RJ). Anais... Volta Redonda (RJ): CASI, 2012. 